\title{
DISCREPANCIES OVER THE ONSET OF SURFACTANT MONOMER AGGREGATION INTERPRETED BY FLUORESCENCE, CONDUCTIVITY AND SURFACE TENSION METHODS
}

\author{
Maria de Fátima Carvalho Costa, Sandro José Froehner, Ângelo Adolfo Ruzza, Sônia de Fátima Santos and Dino Zanette* \\ Departamento de Química, Universidade Federal de Santa Catarina - 88040-900 - Florianópolis - SC
}

Recebido em 2/4/97; aceito em 3/9/97

\begin{abstract}
DISCREPANCIES OVER THE ONSET OF SURFACTANT MONOMER AGGREGATION INTERPRETED BY FLUORESCENCE, CONDUCTIVITY AND SURFACE TENSION METHODS. MOlecular probe techniques have made important contributions to the determination of microstructure of surfactant assemblies such as size, stability, micropolarity and conformation. Conductivity and surface tension were used to determine the critical aggregation concentration (cac) of polymer-surfactant complexes and the critical micellar concentration (cmc) of aqueous micellar aggregates. The results are compared with those of fluorescent techniques. Several surfactant systems were examined, 1-butanol-sodium dodecylsulfate (SDS) mixtures, solutions containing poly(ethylene oxide)-SDS, poly(vinylpyrrolidone)-SDS and poly(acrylic acid)-alkyltrimethylammonium bromide complexes. We found differences between the cac and cmc values obtained by conductivity or surface tension and those obtained by techniques which use hydrophobic probe.
\end{abstract}

Keywords; 1-butanol/sodium dodecylsulfate mixtures; critical aggregation concentration; polymersurfactant interactions; ketal hydrolyses.

\section{INTRODUCTION}

Amphiphiles, in water, spontaneously organize into dynamic aggregates whose properties, e.g. size and form, depend upon monomer structure and concentration as well as composition of solution ${ }^{1}$. Several chemical and physical methods and techniques have been applied in order to explore effects of additives such as salts and alcohols to estimate properties such as the critical aggregation concentration, aggregation number, degree of ionization and ion-exchange constants.

Fluorescent probe techniques have been largely employed for monitoring the formation of surfactant aggregates, principally for two reasons: (i) they are sensitive at a very low probe concentration, and (ii) the fluorescence spectrum can be affected by solvent polarity. Fluorescent probes are, therefore, used to determine the critical micellar concentration of micelles and micellelike aggregates such as polymer-surfactant complexes ${ }^{2}$.

In this work, we have given particular attention to surfactant aggregation in complexes formed between polymer and surfactant. Basically, two fluorescence methods were applied for this purpose. The first was simply to add the probe to the solution containing the surfactant, with or without additives. In this case, there are questions regarding (i) incorporation of the probe at the onset of surfactant aggregate formation; (ii) the use of low concentrations in order to avoid perturbation of the system, and (iii) probe location in the aggregate. To satisfy the first and second requirements, it is only necessary that the probe should be hydrophobic, and solubilization is usually assumed to occur in the Stern layer or at the interface with the hydrophobic micellar core.

Alternatively for polymer-surfactant systems, one prepares the polymer with a covalently-bound probe. In this case, the fluorescent probe detects a microenvironment at the polymer surface and it need not be extremely hydrophobic. However, in both approaches a serious problem is that the hydrophobic probe may perturb the properties of the system, e.g., by inducing aggregation. In the context of fluorescent probe techniques, in both static and dynamic fluorescence modes, pyrene is widely used mainly because of its extreme sensitivity to solvent polarity. The ratio of the fluorescence intensities of vibrational bands $I_{1}$ and $I_{3}$ is indicative of polarity changes, and has been intensively utilized to investigate surfactant assemblies $^{2}$.

In this work, we report a number of results with the goal of estimating an important property of surfactant assemblies, the critical micellar concentration $(\mathrm{cmc})$ for micelles or the critical aggregation concentration (cac) for polymer-surfactant complexes. Use is made of static fluorescence of pyrene, with plots of $I_{3} / I_{1}$ vs. $\log$ sodium dodecyl sulfate concentration, [SDS], and measurements of conductivity and surface tension.

We report results of different surfactant systems and discuss values of cac and $\mathrm{cmc}$, based on conductivity and surface tension methods, comparing them with those from fluorescence methods which use hydrophobic probes such as pyrene. By using kinetic probes, we also show that premicelles form under appropriate conditions. For instance, we report a set of observed first-order rate constant $\left(\mathrm{k}_{\mathrm{obs}}\right)-$ [SDS] profiles for the acid hydrolyses of 2-(p-alkoxyphenyl)-1,3-dioxolanes (alkyl = Me, $\left.n-\mathrm{C}_{6} \mathrm{H}_{13}, \mathrm{n}-\mathrm{C}_{9} \mathrm{H}_{19}\right)$.

\section{EXPERIMENTAL}

Materials. Sodium dodecyl sulfate (SDS), $99 \%$, and dodecyltrimethylammonium bromide (DoTAB) were supplied by Sigma and were used as received. Decyltrimethylammonium bromide (DeTAB) was prepared in a pressure vessel of stainless steel from trimethylamine and n-decyl bromide at $80^{\circ} \mathrm{C}$. The mixture was left for 10 hours in order to react, and was then purified by recrystallization in ethanol-acetone. 2-(p-Methoxyphenyl)-1,3dioxolane (p-MPD) and 2-(p-Nonoxyphenyl)-1,3-dioxolane (p-NPD) were used as described in previous investigations ${ }^{3-5}$. 2-(pHexoxyphenyl)-1,3-dioxolane (p-HPD) was prepared by the procedure of Fife and $\mathrm{Jao}^{6}$. The substrate 2,4-dinitrophenyl octanoate (DNPO) is also described in our previous work ${ }^{7 \mathrm{a}}$. Poly(ethylene oxide) (PEO) and poly(vinylpyrrolidone) (PVP), weight-average molecular weights 10000 and 30000 , respectively, were obtained from Aldrich and were used as received. Poly(acrylic acid) (PAA) 25\% aqueous solution, weight-average molecular weight 90 000, was obtained from Polyscience Inc. The stock solutions of polymers were magnetically stirred for at least 
12 hours: the concentrations are given as molarity on a monomer basis (moles of monomer per liter of solution). The $\mathrm{pH}$ of PAA solutions was carefully adjusted with $\mathrm{NaOH}$. Distilled water was demineralized in a Millipore Milli-Q Water System.

Methods. Fluorescence intensity measurements were made using an SLM AMINCO SPF 500C spectrophotometer (equipped with a thermostated cell-holder set at $25.0^{\circ} \mathrm{C}$ ). Static fluorescence data were used to determine the critical micellar concentration of mixtures of SDS and n-butanol in $0.010 \mathrm{M}$ borate buffer, $\mathrm{pH}=9.10$. The pyrene concentration, $1.6 \times 10^{-6} \mathrm{M}$, was sufficiently low to prevent excimer formation. The slits selected were $5 \mathrm{~mm}$ for excitation and $4 \mathrm{~mm}$ for emission, respectively. All experiments were performed in a solution cell of $10 \mathrm{~mm}$ path length and samples were excited at $330 \mathrm{~nm}$. Solutions of 1butanol were routinely prepared under magnetic stirring for at least 12 hours before use.

Ketal hydrolyses were performed by adding $3 \mu \mathrm{L}$ of substrate stock solution $\left(2 \times 10^{-3} \mathrm{M}\right.$ in dry acetonitrile) into $2.5 \mathrm{ml}$ of $0.02 \mathrm{M}$ succinate buffer, $\mathrm{pH} 5.80$, at $50.0{ }^{\circ} \mathrm{C}$. Benzaldehyde formation was followed spectrophotometrically at $284 \mathrm{~nm}$ by use of a Hewlett-Packard 8452A diode array spectrophotometer. Also, hydrolysis of DNPO was followed spectrophotometrically, at $353 \mathrm{~nm}$ in $0.020 \mathrm{M}$ borate buffer, $\mathrm{pH} 8.75$, in the presence and absence of $1 \mathrm{~g} / \mathrm{L}$ PAA, at $35.0^{\circ} \mathrm{C}$. Rate constants were estimated using HP 89532K kinetic software. Standard deviations for $\log \left(A_{\infty}-A_{t}\right)$ vs. time were less than $10^{-5}$.

Conductance measurements were carried out at 25.0 and $50.0{ }^{\circ} \mathrm{C}$ in a water-jacketed flow dilution cell, with an Analion conductivity meter, model C-701. Stock solutions of mixture of SDS and 1-butanol were routinely prepared under magnetic stirring for at least 12 hours prior to titration. Conductivity data were stored in a microcomputer by use of a Microquímica 12-bits A/D board (Microquímica, Florianópolis, Brazil). The software permits the taking of up to 2,000 points within the concentration range used for titration, and also to amplify surfactant concentration ranges in order to identify particular transition points. Slopes of the conductivity vs. surfactant concentration plots, above and below the experimental breakpoints, were obtained using a standard linear regression routine. Values of cmc, critical aggregation concentration (cac) and polymer saturation point (psp) were defined by the intersection points of these lines.

Surface tension measurements were carried out using a Microquimica MQ-ST1 Surface Tensiometer, employing a drop-weight method. The cac and psp values are estimated by the intersections of the two linear portions of surface tension vs. $\log [\mathrm{SDS}]$ plots above and below the breakpoint points.

\section{RESULTS AND DISCUSSION}

Conductivity Measurements. Figure 1 shows changes in electrical conductivity with added SDS in the presence of 0.001 and $0.010 \mathrm{M}$ PEO. Unlike conventional conductivity profiles in the absence of polymer (see Fig. 3 below), in the presence of polymer the profiles show two breakpoints corresponding to the cac and psp. According to accepted interpretation of conductivity vs. [surfactant] profiles, the cac is attributed to the onset of cooperative adsorption of surfactant on the polymer; the second breakpoint marks the saturation of polymer and, above this [SDS], regular SDS micelles form ${ }^{8-14}$.

We note here, and throughout the text, that the psp indicates only the [SDS] where there is the second breakpoint in conductivity or surface tension profiles. The meaning of the psp breakpoints given in the plots in figure 1 has been discussed previously ${ }^{15}$. Moreover, salt effects on the psp and cac for PEOSDS systems suggest that a real psp is observed only at [PEO] greater than $0.05 \mathrm{M}$. The non-linearity of the plots between the two conductivity breakpoints has also been discussed previously ${ }^{15}$.

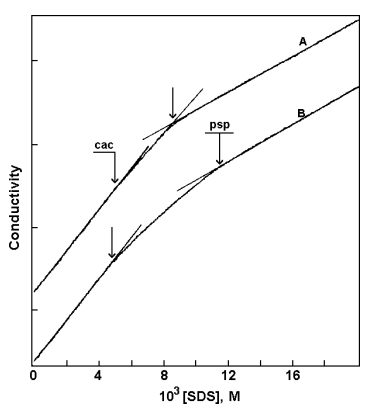

Figure 1. Plots of conductivity (relative scale units) vs. [SDS] in the presence of (A) $1 \times 10^{-3} \mathrm{M}$ and (B) $1 \times 10^{-2} \mathrm{M}$ PEO.

Our reason for focusing on the conductivity vs. [SDS] plots is to emphasize the onset of surfactant binding to polymer, and to show that, below this point, there are questions regarding the existence of pre-aggregates or micelle-like assemblies based on the linearity in the conductivity vs. [surfactant] plots. Figure 1 shows that below cac the plot is linear, indicating no measurable aggregation by this method. The same evidence has been previously related for PEO-SDS ${ }^{4,15}$ and PVP-SDS ${ }^{4}$ systems.

Pyrene $I_{1} / I_{3}$ vibronic peak ratios. The pyrene $I_{1} / I_{3}$ ratio vs. [Surfactant] plot has been extensively used to estimate the $\mathrm{cmc}$ and $\mathrm{cac}^{2,16-24}$ and the extent of penetration of water in micelles, and also of cosurfactants such as alcohols in comicelles ${ }^{25}$. The binding of the probe to surfactant aggregates is generally followed by large changes in fluorescence, providing a convenient method for determining the cmc. Values are sometimes in good agreement with those determined by other methods. Pyrene $I_{1} / I_{3}$ ratios have also been correlated with the efficiency of excimer formation ${ }^{17,22,26}$.

Turro et al. ${ }^{19}$ have applied this technique in polymersurfactant interaction studies for PEO-SDS and PVP-SDS systems. According to their interpretations, the cac value for PEO or PVP is defined by the onset of the plateau in the $I_{1} / I_{3}$ vs. [SDS] plots, and not by the onset of $I_{1} / I_{3}$ changes at a lower [SDS]. Therefore, compared to the conductivity plots, their method of cac determination leads to a debatable interpretation of the profiles. For instance, they found cac values of ca. $2 \times 10^{-3} \mathrm{M}$ and $3 \times 10^{-3} \mathrm{M}$ SDS for PVP-SDS and PEO-SDS systems, respectively. These values disagree with those shown for PEO-SDS (Fig. 1), and for PVP-SDS, cac = $5.0 \times 10^{-3} \mathrm{M}$ (not shown). Moreover, for $0.02 \%$ PEO (i.e. 4.5 $\mathrm{x} 10^{-3} \mathrm{M}$ on a monomer basis) they noted that the profile showed only one break at ca. $7 \times 10^{-3} \mathrm{M}$ SDS, therefore, the data did not show the lower breakpoint.

We are able to explain these observations, basing our argument on the conductivity and surface tension measurements previously reported ${ }^{15}$. First, we demonstrated that the second conductivity breakpoint at low polymer concentrations, i.e., below $5 \times 10^{-3} \mathrm{M}$, is driven by formation of SDS micelles. Figure 1 shows the conductivity vs. $[\mathrm{SDS}]$ profile at $[\mathrm{PEO}]=1.0 \times 10^{-3} \mathrm{M}$, and a best psp estimation gives $8.4 \times 10^{-3} \mathrm{M}$, agreeing with the cmc of SDS reported elsewhere ${ }^{27}$. Secondly, below $[\mathrm{PEO}]=5 \times 10^{-3} \mathrm{M}$, the ratios of the slopes, which are related to the degree of ionization of PEO-SDS complexes, taken above and below the cac, increase towards unity. This means that the accuracy of the predicted cac becomes lower. According to this analysis, it is not surprising that in the $\mathrm{I}_{1} / \mathrm{I}_{3}$ vs. [SDS] plots in $0.02 \%$ PEO the first breakpoint is not observed. Commenting on this work, Goddard ${ }^{10}$ stated that there was no explanation for the onset of $I_{1} / I_{3}$ reduction starting at very low SDS concentration $\left(\sim 10^{-3} \mathrm{M}\right)$, i.e., lower than the cac.

In estimation of the cac or cmc using the pyrene $I_{1} / I_{3}$ method, it is considered that at the onset of surfactant aggregation pyrene should be almost totally bound to surfactant 
aggregates. The ratio $I_{1} / I_{3}$ changes up to a limiting value, usually for anionic surfactant, between 0.9 and 1.3 , but the shape of the described isotherms (in the $\mathrm{I}_{1} / \mathrm{I}_{3}$ vs. [SDS] method) depends on the surfactant and on the composition ${ }^{16,19-21,24,28}$. The main question now becomes that of establishing the correct methodology for defining cac or cmc. For instance, Maltesh and Somasundaram ${ }^{16}$ selected the SDS concentration corresponding to the onset of isotherm saturation at which $\mathrm{I}_{3} / \mathrm{I}_{1}$ is $\approx 1$. However, for the PEO-SDS system, in the presence of $20 \mathrm{ppm}$ PEO, they defined cac as the SDS concentration at which the interactions between PEO and SDS commence ${ }^{26}$. Their argument was supported by the fact that the surface tension vs [SDS] plot indicated a transition point at the same [SDS]. We note that this methodology differs from that used by Turro et al. ${ }^{19}$ In another work, Turro and $\mathrm{Lei}^{28}$ defined the $\mathrm{cmc}$ from $I_{3} / I_{1}$ vs. [SDS] plots in the presence and absence of buffer at a concentration close to the isotherm saturation.

The cmc in mixtures of 1-butanol and SDS. The aim of this topic is to draw attention to the uncertainty of the definition of the onset of surfactant aggregation in 1-butanol-SDS mixtures using the $I_{3} / I_{1}$ vs. [SDS] plots. We choose this particular system as a practical example of discrepancy between the above and conductivity techniques.

Typical changes in the polarity parameter $I_{3} / I_{1}$ of pyrene are shown in figure 2 , in $0.010 \mathrm{M}$ borate buffer, $\mathrm{pH}=9.10$. The $\mathrm{I}_{3} / \mathrm{I}_{1}$ ratio vs. [SDS] plots are monitored in mixtures of 1-butanol and SDS to determine the role played by the alcohol in the critical micellar concentration.

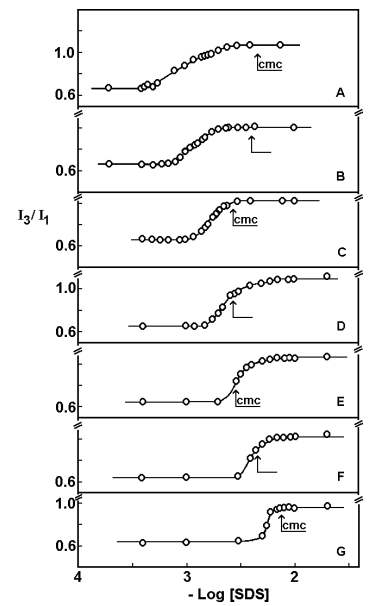

Figure 2. Ratio of third to first vibrational peak of pyrene emission spectrum as a function of $\log [S D S]$, in $0.010 \mathrm{M}$ borate buffer, $p H=9.10$, at the following 1-butanol concentrations: (A) $0.60 \mathrm{M} ;(B) 0.50 \mathrm{M} ;(C) 0.40 \mathrm{M}$; (D) $0.30 \mathrm{M} ;(E) 0.20 \mathrm{M} ;(F) 0.10 \mathrm{M}$ and $(G)$ in the absence of 1-butanol, at $25.0^{\circ} \mathrm{C}$. The arrows indicated the cmc measured by conductivity vs $[S D S]$ profiles (from Fig. 3) under the same experimental conditions.

It is well recognized that micellar incorporation of moderately hydrophobic alcohols decreases the micellar charge density, resulting in an increase in the degree of ionization and a decrease in aggregation number ${ }^{25}$. The accuracy of $\mathrm{cmc}$ or cac measurements is important to the estimation of size based on fluorescence quenching methods. They relate the calculation of aggregation number, $n$, to the concentration of surfactant aggregates present in solution. For steady fluorescence quenching method $^{29}, n$ can be estimated from eq. (1), where Cd refers to

$n=\mathrm{Cd} /[$ Micelles $]$

concentration of surfactant aggregates, related to total surfactant concentration, $\mathrm{Ct}=\mathrm{Cd}+\mathrm{cmc}$ or $\mathrm{Ct}=\mathrm{Cd}+\mathrm{cac}$, in polymer- surfactant mixtures. In this context, the $\mathrm{cmc}$ or cac are important parameters necessary to estimation of the aggregation numbers.

It may be clearly observed in figure 2 that the isotherms are shifted to a lower [SDS], as is expected for the effect of 1-butanol on the $\mathrm{cmc}^{25}$. For comparison, figure 3 shows a set of conductivity vs. [SDS] plots obtained under conditions similar to those used to obtain the data in figure 2 . In the absence of 1-butanol, the value of $\mathrm{cmc}\left(6.70 \times 10^{-3} \mathrm{M}\right)$ obtained by conductivity is identical to the SDS concentration at the onset of the plateau of the $I_{3} / I_{1}$ vs. [SDS] plot (Fig. 2). This close correspondence between the two methods is not general. Therefore, the arrows in figure 2 indicate $\mathrm{cmc}$ values measured by conductivity. According to the conductivity method, the inflection point in the $\mathrm{I}_{3} / \mathrm{I}_{1}$ vs. [SDS] plots suggested by the literature $\mathrm{e}^{21-23}$ is a reasonable choice only in the presence of certain concentrations of alcohol. But at 0.5 and $0.6 \mathrm{M}$ 1-butanol, the conductivity values correspond to [SDS] beyond the onset of the saturation.

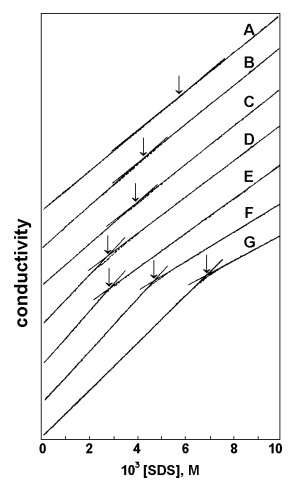

Figure 3. Plots of conductivity (relative scale units) vs. [SDS] in 0.010 $M$ borate buffer, $p H=9.10$, at the following 1-butanol concentrations: (A) $0.80 \mathrm{M}$; (B) 0.60 ; (C) $0.50 ;(D) 0.30 ;(E) 0.20 ;(F) 0.01$ and $(G)$ in the absence of 1-butanol, at $25.0^{\circ} \mathrm{C}$.

The cac at low PEO concentration. Recently, Maltesh and Somasundaram ${ }^{26,30}$ have used PEO end-labeled with pyrene at $20 \mathrm{ppm}$ (which corresponds to $0.455 \mathrm{mM}$ monomer units) to monitor changes in polymer conformation due to association with SDS. Measurements were carried out at a very dilute [PEO], to ensure that all aggregates were bound to the polymer and that there were no free micelles. By fluorescence spectroscopy, they determined the effect of various salts on the binding. The authors chose to interpret the fluorescence data, taking the initial surfactant binding to the polymer, cac, as the onset of changing monomer intensity and excimer emission ratios $\left(\mathrm{I}_{\mathrm{E}} / \mathrm{I}_{\mathrm{M}}\right)$ plotted against the surfactant concentration.

On the other hand, Winnik et al. ${ }^{17}$, studying an analogous system, interaction of SDS with a PEO containing pyrene groups at both ends attached via ether linkages, concluded that pyrene groups act cooperatively to promote interaction between SDS and the polymer. The changes in emission should be due to interactions between hydrophobic pyrene groups and the surfactant. Lissi et al. ${ }^{31}$ explored the same system, but at PEO concentrations higher than those described in refs. 26 and 30. According to these authors, in disagreement with Maltesh and Somasundaram, the critical aggregation concentration closely corresponds to the maximum value of $\mathrm{I}_{\mathrm{E}} / \mathrm{I}_{\mathrm{M}}$ plotted against surfactant concentration, and they suggest that the far higher hydrophobicity of a pyrene moiety relative to that of the oxyethylene units makes the polymer chain ends the best binding sites for the surfactant and, therefore, the interaction starts by adsorption of the surfactant at the chain ends.

We recently used conductivity and surface tension vs [SDS] plots, at low PEO concentration, in order to follow the effect of salt on the cac and psp breakpoints ${ }^{15}$. In spite of the 
low [PEO] condition, the surface tension and conductivity plots still exhibit profiles similar to those seen at higher concentrations $3,7,9,11,14$. Furthermore, at SDS concentrations above the cac, the surface tension vs. [SDS] plots (see ref. [15]) exhibit narrow plateaus, and then decrease. These plateaus of constant monomer activity could be attributed to an [SDS] range where only polymer-surfactant complexes are forming. This argument was used by Maltesh and Somasundaram ${ }^{26}$. Table 1 summarizes the cac and psp values determined by surface tension vs. [SDS] plots in the presence of $0.5 \mathrm{mM}$ PEO and at different $[\mathrm{NaCl}]$. These values are plotted in figure 4 . The plots are linear and obey eqs. (2) and (3) for cac and cmc, respectively.

$\log \mathrm{cac}=-0.71 \log (\mathrm{cac}+[\mathrm{NaCl}])-3.99$

$\log \mathrm{cmc}=-0.78 \log (\mathrm{cmc}+[\mathrm{NaCl}])-3.59$

Table 1. Values of first (cac) and second (psp) breakpoints determined by surface tension vs. [SDS] plots for $[\mathrm{PEO}]=0.5 \mathrm{mM}$, at different $\mathrm{NaCl}$ concentrations and at $25.0^{\circ} \mathrm{C}$.

\begin{tabular}{ccc}
\hline $10^{3}[\mathrm{NaCl}], \mathrm{M}$ & $10^{3} \mathrm{cac}, \mathrm{M}$ & $10^{3} \mathrm{psp}, \mathrm{M}$ \\
\hline None & 4.50 & 9.30 \\
10 & 2.51 & 6.30 \\
50 & 1.26 & 2.82 \\
100 & 0.55 & 2.19 \\
200 & 0.26 & 1.00 \\
500 & 0.18 & 0.40 \\
$500^{\text {(a) }}$ & - & 0.54 \\
\hline
\end{tabular}

(a) In the absence of PEO

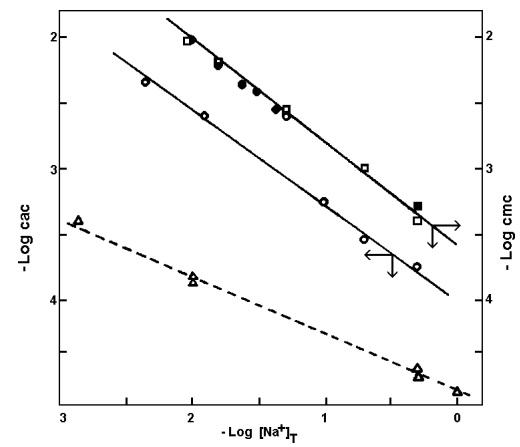

Figure 4. Plots of $\log \left[\mathrm{Na}^{+}\right]_{T}$ versus: (•) log $\mathrm{cmc}$ for SDS in the absence of PEO obtained by conductivity plots from Ref.[15]; (口) log of the second breakpoint ( $p s p$ ) in the presence of $0.5 \mathrm{mM} P E O$ (from Table 1); (-) log cmc for SDS in the absence of PEO, at $0.5 \mathrm{M} \mathrm{NaCl}$ (from Table 1); (O) log cac in the presence of $0.5 \mathrm{mM}$ PEO obtained by surface tension plots (from Table 1); $(\Delta) \log$ cac in the presence of 20 ppm PEO from Ref. [26] (see text).

For comparison, figure 4 also shows the cmc values (in the absence of PEO) obtained from conductivity vs [SDS] profiles reported earlier ${ }^{15}$, except at $[\mathrm{NaCl}]=0.5 \mathrm{M}$ (black square) which was obtained by surface tension. We note that the slopes of $\log$ psp (which in Fig 4 is $\log \mathrm{cmc}$ ) vs. $\log \left[\mathrm{Na}^{+}\right]$are very similar, providing strong evidence that the second breakpoint in the surface tension vs $\log$ [SDS] profiles is driven by formation of SDS micelles, and therefore it is a major piece of evidence that here the psp are related to formation of SDS micelles and not to saturation of the polymer by surfactant, as conventionally described $8,10,12,14$.

We also show in figure 4 plots of the values of cac from ref. 30. The slope is -0.44 . We note that the obtained linear relationship shifts from that given by surface tension. These significant differences between the cac plots can also be discussed in terms of the degree of ionization of PEO-SDS complexes, such as that of SDS micelles ${ }^{32,33}$, where it is assumed that the slope is equal to $(1-\alpha)$. In the above cases, the method of slopes provides $\alpha$ values of 0.29 for SDS-PEO complexes and ca. 0.66 for the PEO end-labeled pyrene-SDS system $^{26,30}$. The differences are significant as compared to $\alpha=0.22$ obtained in this work for SDS micelles.

Finally, we conclude that the hydrophobic pyrene moiety induces pre-aggregation and the fluorescence data determined by using the ratio $\left(\mathrm{I}_{\mathrm{E}} / \mathrm{I}_{\mathrm{M}}\right)$ can be incorrectly attributed to the surfactant-polymer complexes. This is for two reasons: i) In the surface tension vs. $\log$ [SDS] profiles, we could not observe any kind of discontinuity in values of [SDS] corresponding to the cac obtained from the $\mathrm{I}_{\mathrm{E}} / \mathrm{I}_{\mathrm{M}}$ ratio (not shown); ii) the $\alpha$ value of 0.66 obtained by the method of slopes (Fig. 4) is too large compared to that of 0.29 obtained by the same method; usually, high $\alpha$ values are typical of small aggregates. For instance, it is well documented that adding alcohols such as 1-butanol to SDS increases $\alpha$ as the aggregation number decreases ${ }^{25,34}$.

Kinetic probes. Premicelles are often identified by kinetic probes $^{7 \mathrm{a}, 35}$. For instance, the inhibition of hydrolyses of 2,4-dinitrophenyl acetate and benzoic anhydride at low sodium dodecanoate concentrations was recently interpreted by assuming the formation of kinetically ineffective premicellar complexes ${ }^{7 \mathrm{a}}$.

Figure 5A shows in detail, at low [SDS] and in the presence of $0.5 \mathrm{mM}$ PEO, the biphasic profiles for the effect of [SDS] on the observed first-order rate constant for acid hydrolyses of the 2-(p-alkoxyphenyl)-1,3-dioxolanes (Scheme 1). The kinetic runs were carried out at $50.0^{\circ} \mathrm{C}$ to follow the p-NPD hydrolysis close to the cac, because of its low solubility in water. Part of the rate profile, represented by the dashed lines, was extrapolated to water assuming the same reactivity of $\mathrm{p}$-MPD and the other dioxolanes.

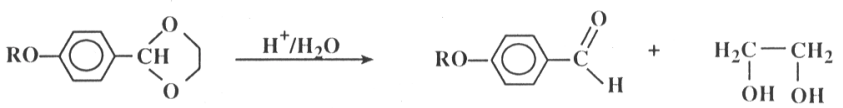

p-MPD, $\mathrm{R}=\mathrm{CH}_{3} ; \mathrm{p}-\mathrm{NPD}, \mathrm{R}=\mathrm{C}_{9} \mathrm{H}_{19} ; \mathrm{p}-\mathrm{HPD}, \mathrm{R}=\mathrm{C}_{6} \mathrm{H}_{13}$

Scheme 1

Recently, we reported catalysis of acid hydrolysis of pNPD below the cac as shown by the $\mathrm{k}_{\mathrm{obs}}-\left[\mathrm{SDS}\right.$ ] profile ${ }^{4}$. In this work, discontinuities observed in the kinetic profile coincided with the breakpoints of the specific conductivity vs. [SDS] plots. To interpret these kinetic profiles as evidence of polymer-surfactant interactions, figure 5B shows the conductivity vs. [SDS], under the experimental conditions described in figure 5A. The profile exhibits the typical behavior of that shown in figure 1 .

Qualitatively, figure 5A depicts three distinct kinetic regions. For p-MPD the rate increases only slightly in the SDS concentration range between the cac and psp. The cac breakpoint is not identified by the probe. However, the rate clearly increases above the psp. For p-HPD, the rate begins its increase at the cac determined by conductivity (Fig. 5B). Below this point, $\mathrm{k}_{\mathrm{obs}}$ is constant, as in water for $\mathrm{p}$-MPD. In contrast, we cannot say the same for $\mathrm{p}-\mathrm{NPD}$. At the cac, $\mathrm{k}_{\mathrm{obs}}$ differs by a factor of 5 from that of p-HPD. Unfortunately, below this [SDS] we could not estimate the rate constant because solutions were cloudy. We assume that in water the reactivity of p-NPD should be similar to that of the other substrates which have the phenyl-1, 3-dioxolane moiety.

Polyelectrolyte-cationic surfactant system. Fluorescent molecules are also extensively used to probe the surfactant environments in the presence of an oppositely-charged polymer. 


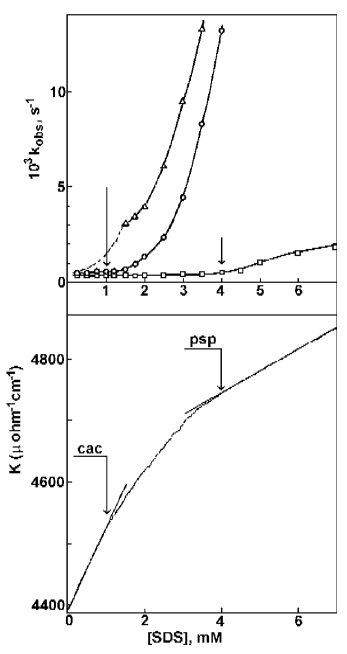

Figure 5. (A) Effect of increasing SDS concentration on the $k_{o b s}$ for acid-catalyzed hydrolysis of $(\Delta) p-N P D$, ( ( ) $p-H P D$ and (口) $p-M P D$, in 0.020 succinate buffer, $p H=5.80$, at $50.0{ }^{\circ} \mathrm{C}$, in the presence of $0.5 \mathrm{mM}$ PEO; (B) conductivity vs. [SDS] plot in 0.020 succinate buffer, $p H=5.80$, at $50.0^{\circ} \mathrm{C}$, in the presence of $0.5 \mathrm{mM} \mathrm{PEO}$. The dashed lines in Fig. 8A are extrapolated.

The surfactant is able to associate to polymer, owing to electrostatic and hydrophobic factors, and the polymer is able to induce micelle-type aggregation at surfactant concentrations lower than the cmc. Fluorescence quenching methods, timeresolved quenching and steady-state fluorescence quenching, have been largely used to study the behaviors of polyeletrolyte in the presence and absence of surfactant $t^{2,19,22-24,28}$. These methods provide information on polymer conformations and on interactions with surfactant and additives: the most common parameter determined is the aggregation number.

In a recent review, Lindman and Thalberg ${ }^{36}$ made significant comments on different aggregation behavior reported for different polyeletrolyte-surfactant systems. They noted the large aggregation number of $105 \pm 10$ obtained by Chu and Thomas ${ }^{24}$ for poly(methacrylate) (PMA) and DeTAB. They suggested that further systematic studies would be required concerning different parameters and also concerning the relation between the degree of binding and the aggregation number.

Thalberg et al. ${ }^{37}$, investigating polyelectrolyte-surfactant interactions, in particular interactions of the anionic polysaccharide hyaluronate, and decyl (DeTAB) and dodecyl (DoTAB) trimethylammonium bromide, justified their proposals by stating that the few studies performed on these types of systems gave a rather scattered picture. The authors referred to overestimation of the aggregation numbers of poly(methacry latic acid) (PMA) and DeTAB ${ }^{24}$, in part because they included in the calculation the free monomer concentration. However, questions arise regarding the cac value, since the accuracy of aggregation numbers depends on the surfactant concentration used in the experiments. That is, for measurements at a large $\mathrm{Cd}$, small shifts in cac do not significantly affect the results. However, this is not so for experiments performed close to the cac. Chu and Thomas ${ }^{24}$ measured the aggregation number at 8 $\mathrm{x} 10^{-3} \mathrm{M}$ DeTAB and determined a cac $=3 \times 10^{-3} \mathrm{M}$ by considering the surfactant concentration corresponding to a steady value of pyrene $\mathrm{I}_{3} / \mathrm{I}_{1}$ vs. [DeTAB] plots, at $\mathrm{pH}=8$ and $1 \mathrm{~g} / \mathrm{L}$ polymer.

However, figure 6 shows conductivity vs [DeTAB] and [DoTAB] plots for $1 \mathrm{~g} / \mathrm{L}$ poly(acrylic acid), at $\mathrm{pH}=8.75$ and 6.40 , where values of cac are $5.25 \times 10^{-3} \mathrm{M}$ and $3.15 \times 10^{-4} \mathrm{M}$, respectively. For DeTAB, the cac is not very different from that obtained by $\mathrm{Chu}$ and Thomas ${ }^{24}$, although they used poly (metacrylate), but it is also very similar to a cac $=3.63 \mathrm{x}$ $10^{-3} \mathrm{M}$, recently reported for poly(acrylic acid) and DeTAB ${ }^{23}$. However, if the aggregation number were estimated under their experimental conditions, the calculated aggregation number should be ca. 31 , considering the cac found in our work. But using their cac value, the aggregation number corresponds to 68 . This newly calculated value $(\mathrm{N}=31)$ is in the same range as those obtained by Thalberg et al. ${ }^{37}$ for the hyaluronateDeTAB system. For poly(acrylic acid)-DoTAB system, $\mathrm{pH}=9$, we note that Chandar et al. ${ }^{22}$ estimated cac $\cong 1.0 \times 10^{-4}$ $\mathrm{M}$, which is moderately lower than that obtained by conductivity in this work.

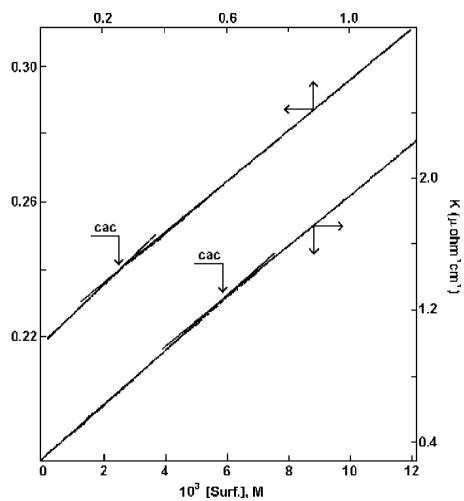

Figure 6. Conductivity vs. [Surfactant] profiles for (A) DeTAB at $p H=8.75$ and $(B)$ DoTAB at $p H=6.40$ of solutions with $1.0 \mathrm{~g} / \mathrm{L}$ $P A A$, at $25.0{ }^{\circ} \mathrm{C}$.

An additional significant conclusion is related to the ratio of the slope in the conductivity vs. [surfactant] plots, above and below cac (Fig. 6). Since the ratios are related to the degree of dissociation of the complex, in both cases ca. 0.90, in a first approximation they agree with the fact that the polymer aggregates are small. For instance, the effect of 1-butanol on the conductivity ratios reported here (Fig. 3) and elsewhere ${ }^{25}$ is in agreement with the formation of small aggregates.

Supporting the above comments, figure 7 shows $k_{o b s}$ [DeTAB] profiles for hydrolysis of a hydrophobic substrate, the 2,4-dinitrophenyl octanoate (DNPO) at $0.02 \mathrm{M}$ borate buffer, $\mathrm{pH}=8.75$, in the presence and absence of $1 \mathrm{~g} / \mathrm{L}$ PAA. The rate maxima observed in $\mathrm{k}_{\mathrm{obs}}$ just above cac and $\mathrm{cmc}$, in the presence and absence of PAA, respectively, are typical of micellar catalyzed bimolecular reactions ${ }^{1,5,32}$. However, the differences in maximum $\mathrm{k}_{\mathrm{obs}}$ values, ca. 4-fold, are interpreted as differences in "local" $\mathrm{OH}^{-}$concentrations in the micellar or PAA-DeTAB complex interfaces where the substrate reacts. Moreover, in the pseudophase ion exhange (PPIE) model of micellar catalysis ${ }^{1,3-5,32}$ the degree of ionization is an important parameter in simulation of the $\mathrm{K}_{\mathrm{obs}}$ - [SDS] profiles, and $\alpha$ has a significant effect on the concentration of bound $\mathrm{OH}^{-}$and, consequentely, on the DNPO hidrolyis rate. Thus, for this particular kind of reaction, aggregates with large micellar coverage (low $\alpha$ ) effectively concentrate $\mathrm{OH}^{-}$, and this is an important factor in the interpretacion of enhancements. Finally, DNPO, bound to both aggregates, detects the "local" $\mathrm{OH}^{-}$concentration differently, and this fact leads us to conclude that DeTAB micelles are better catalysts than PAA-DeTAB complexes. The same conclusion was reached for acid-catalysed acetal hydrolysis in solutions containing PEO or PVP and $\mathrm{SDS}^{3,4}$. 


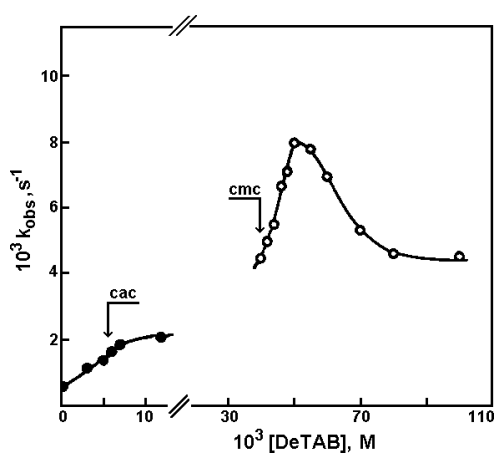

Figure 7. Effect of SDS on $k_{\text {obs }}$ for hydrolysis of DNPO in $0.020 \mathrm{M}$ borate buffer, $\mathrm{pH}=8.75$, in presence $(\bullet)$ and absence $(0)$ of $1 \mathrm{~g} / \mathrm{L}$ $P A A$, at $35.0^{\circ} \mathrm{C}$. Values of cac are taken from Fig. 6. Lines are drawn to aid the eye.

\section{CONCLUSION}

Fluorescence probe methods give important information on the microstructure of the polymer-surfactant complexes. However, discrepances can be found in results of micellar properties in which the knowledge of the parameter cmc or cac is necessary. Examples are in the estimations of aggregation number (see eq. (1)) and free energies. For example, free energies have been estimated using $\Delta \mathrm{G}_{\mathrm{cac}}=\mathrm{RT} \ln (\mathrm{cac})^{9,23,24}$. It is expected that values depend on the accuracy of cac. It has been documented that fluorescent studies with free pyrene and pyrene-labeled PAA lead one to conclude that cac, determined by eximer formation of a covalently-labeled polymer, is different from that measured from the micropolarity parameter $\left(\mathrm{I}_{3} / \mathrm{I}_{1}\right)$ vs. [DoTAB] plots ${ }^{22}$. This difference is attributed to a hydrophobic contribution to the binding process resulting from the presence of pyrene label which decreases surfactant concentrations for aggregation.

More recently, Bloor et al. ${ }^{38}$ reported binding of sodium alkyl sulfates to a polymer labeled with a covalently-bonded solvatochromic probe, and, from analysis of the spectral data, they concluded that these aggregates preferentially bind to the chromophore because the interpretation of spectroscopic measurements as a function of [SDS] did not agree with the critical aggregation concentration values found by electromotive force (emf) and isothermal titration calorimetry (ITC) methods.

It is relevant to emphasize that the surfactant systems examined in this work, 1-butanol-sodium dodecylsulfate (SDS) mixtures, poly(ethylene oxide)-SDS and poly(vinylpyrrolidone)SDS mixtures, and poly(acrylic acid)-alkyltrimethylammonium bromide complexes, show significant differences between the cac and cmc values obtained by conductivity or surface tension and those obtained by techniques which use hydrophobic probe. Therefore, the results and comments reported here concerning uncertainties over the methods employing hydrophobic probes to measure the onset of surfactant aggregation, provide a qualitative evaluation of the relevance and use of these techniques.

\section{ACKNOWLEDGMENT}

We are grateful to CNPq and PRONEX for the financial support of this work. We would also like to thank Prof. Clifford A. Bunton of the NSF U.S.- Latin American Cooperative ProgramBrazil (INT-9102214) for the helpful revision of this manuscript.

\section{REFERENCE}

1. Bunton, C. A.; Nome, F.; Quina, F. H.; Romsted, L. S.; Acc. Chem. Res. 1991, 24, 357.

2. Winnik, F. M.; Regismond, S. T. A.; Colloids Surf. 1996, $118,1-39$
3. Ruzza, A. A.; Froenher, S. J.; Minatti, E.; Nome, F.; Zanette, D.; J. Phys. Chem. 1994, 98, 12361.

4. Zanette, D.; Ruzza, A. A.; Froenher, S. J.; Minatti, E.; Colloids Surfaces 1996, 108, 91.

5. Ruzza, A. A.; Nome, F.; Zanette, D.; Romsted, L. S.; Langmuir 1995, 11, 2393.

6. Fife, T. H.; Jao, L. K.; J. Org. Chem. 1965, 30, 1492.

7. (a) Marconi, D. M. O.; Frescura, V. L. A.; Zanette, D.; Nome, F.; Bunton, C. A.; J. Phys. Chem. 1994, 98, 12415; (b) Frescura, V. L. A.; Marconi, D. M. O.; Zanette, D.; Nome, F.; Bunton, C. A.; J. Phys. Chem. 1995, 99, 11495. 8. Schwuger, M. J.; J. Colloid Interface Sci. 1973, 43, 491.

9. (a) Dubin, P. L.; Gruber, J.; Xia, J.; Zhang, H. J.; J. Colloid Interface Sci. 1992, 148, 35. (b) Xia, J.; Dubin, P. L.; Kim, Y.; J. Phys. Chem. 1992, 96, 6805.

10. Goddard, E. D.; In Interactions of Surfactants with Polymers and Proteins; Goddard, E. D.; Ananthapadmanabhan, K. P., Eds.; CRC Press Inc: BoCa Raton, FL, 1993; pp 123-169.

11. Lissi, E. A.; Abuin, E. J.; J. Colloid Interface Sci. 1985, 105, 1.

12. Murata, M.; Arai, H.; J. Colloid Interface Sci. 1969, 44, 475.

13. Cabane, B.; J. Phys. Chem. 1977, 81, 1639.

14. François, J.; Dayantis, J.; Sabbadin, J.; J. Eur. Polym. 1985, 21, 165.

15. Minatti, E.; Zanette, D.; Colloids Surfaces 1996, 113, 237.

16. Maltesh, C.; Somasundaran, P.; Colloids Surfaces 1992, 69, 167

17. Yong-Zhong, H.; Cheng-Le, Z.; Winnik, M. A.; Langmuir 1990, 6, 880 .

18. Choi, L.-S.; Kim, O.-K.; Langmuir 1994, 10, 57.

19. Turro, N. J.; Baretz, B. H.; Kuo, P. L.; Macromolecules 1984, 17,1321.

20. Winnik, F. M.; Winnik, M. A.; Tazuke, S.; J. Phys. Chem. 1987, 91, 594.

21. Van Stam, J.; Wittouck, N.; Almgren, M.; De Schryver, F. C.; Miguel, M. G.; Can. J. Chem. 1995, 73, 1765.

22. Chandar, P.; Somasundaram. P.; Turro, N. J.; Macromolecules 1988, 21, 950.

23. De Oliveira, V. A.; Tiera, M. J.; Neumann, M. G.; Langmuir 1996, 12, 607.

24. Chu, D.-Y.; Thomas, J. K.; J. Am. Chem. Soc. 1986, $108,6270$.

25. Zana, R.; Adv. Colloid Interface Sci. 1995, 57, 1.

26. Maltesh, C.; Somasundaran, P.; Langmuir 1992, 8, 1926.

27. Mukerjee, P.; Mysels, K. J.; Critical Micelle Concentrations of Aqueous Surfactant System, National Bureau of Standards, Washington, DC, 1971.

28. Turro, N. J.; Lei, X. G.; Langmuir 1995, 11, 2525.

29. Turro, N. J.; Yekta, A.; J. Am. Chem. Soc. 1978, 100, 5951.

30. Maltesh, C.; Somasundaran, P.; J. Colloid Interface Sci. 1993, 157, 14.

31. Quina, F.; Abuin, E.; Lissi, E.; Macromolecules 1990 , $23,5173$.

32. Romsted, L. S.; PH.D. Thesis, Indiana University, Bloomington, IN, 1975.

33. Mukerjee, P.; Mysels, K. J.; Kapauuan. P.; J. Phys. Chem. 1967, 71, 4166.

34. Rubio, A. R.; Zanette. D.; Nome. F.; Bunton, C. A.; Langmuir 1994, 10,1151.

35. Hioka, N.; Politi, M. J.; Chaimovich, H.; Tetrahedron Lett. 1989, 30, 1051 .

36. Lindman, B.; Thalberg, K.; In Interactions of Surfactants with Polymers and Proteins; Goddard, E. D.; Ananthapadmanabhan, K. P., Eds.; CRC Press Inc: BoCa Raton, FL, 1993; pp 203-276.

37. Thalberg, K.; van Stam, J.; Lindblad, C.; Almgren, M.; Lindman, B.; J. Phys. Chem. 1991, 95, 8975.

38. Bloor, D. M.; Li, Y.; Wyn-Jones, E.; Langmuir 1995, 11, 3778. 